\title{
Cure-Recovery -- Research on the Development of Urban Public Art in the Post-epidemic Context
}

\author{
Hao Kaili ${ }^{1, a *}$ Liang Yan ${ }^{2, b}$ \\ ${ }^{1}$ Department of Environmental Design, School of Art and Design, Lanzhou Jiaotong University, Anning, Lanzhou, \\ Gansu, China \\ ${ }^{2}$ Department of Environmental Design, School of Art and Design, Lanzhou Jiaotong University, Anning, Lanzhou, \\ Gansu, China \\ a*1695708419@qq.com \\ b930969@qq.com
}

\begin{abstract}
A novel Coronavirus outbreak has disrupted the development process of all countries in the world. Isolation and distancing have made wearing masks seem to be a sensitive word in the post-epidemic era. Face the dilemma, our senses of the city will be infinite amplification, loneliness, depression, irritability and other emotional will change the way we see the world, even as a "living soul" for the city public art will broaden its art form of expression border and, to a whole new space field, from different aspects such as vision, the spirit of encouraging public, become a part of the art form of final show, Changing their consciousness and reshaping their social memory. Based on the spirit of place, public art will make use of the deceleration opportunity provided by COVID-19 to intervene in the public spirit and special space of the city, and acutely capture the characteristics of construction. Both in content and form, it is a response and collision to the gray period that has been lasting for a long time, and establish a relationship of healing and recovery. This is also the unique mission of urban space construction in the post-epidemic era endowed by the fundamental nature of public art, which guides us out of the haze and heals the urban space through art.
\end{abstract}

Keywords: pre-epidemic, in-epidemic, epidemic normalization, public art development, urban renewal

\section{治愈・复苏一后疫情背景下城市公共艺术现状发展研究}

郝凯利 ${ }^{1,}$ a*梁艳 ${ }^{2, b}$

${ }^{1}$ 兰州交通大学艺术设计学院环境设计系, 安宁, 兰州, 甘肃, 中国

2 兰州交通大学艺术设计学院环境设计系, 安宁, 兰州, 甘肃, 中国

a*1695708419@qq.com

b930969@qq.com

\begin{abstract}
摘要
一场突如其来的新冠病毒打乱了全球各国的发展进程, 封闭、拉开距离, 佩戴口罩似乎成为延续至后疫情时代 的敏感词汇。面对困局, 我们对于所在城市的感官将被无限放大, 孤独、抑郁, 烦躁等情绪甚至会改变我们看 待世界的方式，作为城市 “活的灵魂” 的公共艺术将拓宽其艺术表达边界与形式，转向全新的空间场域，从视 觉、精神等不同层面鼓励大众, 成为艺术最终展现形式的一部分, 改变其意识的同时重塑社会记忆。在此, 公 共艺术将基于场所精神, 借助新冠疫情为城市提供的减速机会, 介入公众精神层面和城市特殊空间, 敏锐捕捉 营造特性，无论是在内容上还是形式上，都是对持续已久的灰色时期的回应与碰撞，建立治愈和复苏关系。这 也是公共艺术的根本属性赋予的对后疫情时代城市空间营造的独特使命, 通过艺术引导我们走出阴霧, 愈合城 市空间。
\end{abstract}

关键词: 疫前, 疫中, 疫情常态化, 公共艺术现状发展, 城市更新 


\section{1. 前言}

2019 年底初次爆发的新冠肺炎席卷全球，在各 个国家加快发展脚步的时候, 疫情的到来无疑给许多 国家地区造成人口、经济等损失。人们进入一种全城 戒备、恐慌、无助直至麻木的状态，作为随着时代应 运而生的公共艺术，在本质上具有干预社会发展，解 决社会困顿的能力。因此, 面对疫情危机带来困局, 我们应转变思路, 从“危” 中见新 “机”，以艺术的 方式助力城市更新发展 ${ }^{[1]}$ 。回看 20 世纪 30 年代, 美 国在经历了经济大萧条后城市一片衰败, 1935 年, 总统罗斯福为使城市重现生机, 一方面推行新政, 另 一方面则特别委派公共事业振兴署（WPA）向艺术家 提供制作大型壁画的工作机会, 组织全国性的作品巡 展, 试图用艺术的方式振兴城市面貌, 帮助人们恢复 信心 ${ }^{[2]}$ 。在此之后, 公共艺术的发展就与城市更新有 着密不可分的关系, 面对疫情的肆虐, 生命的脆弱被 一览无余, 重新考量生存的意义和活着的希望此时看 来比以往更有讨论价值。各国的公共艺术家们再一次 展现公共艺术的力量, 在提升城市居住环境、展现地 域文化、重塑城市内在精神、提高人民艺术修养的同 时用艺术化解疫情留给失落城市空间的阴皬, 为人们 带去色彩和希望, 并试图抚平人们内心的焦躁。公共 艺术是一个城市活的灵魂, 他不是高高在上的, 是凝 聚普罗大众集体记忆的载体, 是促进艺术交流, 人人 都是艺术家, 提供多元、公共、亲民的共生共融的空 间。

\section{2. 新冠疫情对城市公共艺术发展的影响变化}

在经历了 “魔幻世界” 的 2020 年后, 可以说世 界早已千疮百孔, 可能是对这个世界变化还存在一 丝侥幸, 以为就如同非典病毒、鼠疫等一样可以完 全绝杀, 人类在经历这次渡劫中也会战胜新冠疫情。 然而, 从 2019 年底到 2020 一整年, 在到 2021 年快 接近尾声, 近两年过去了, 我们似乎已经习惯了和 人产生距离, 与口罩共存, 且至今未能成功的找到 破解疫情的源头方法。

随着疫情短期内常态化, 环顾整个社会生态自 然, 河南和山西等地接连被洪水淹没, 北方的夏季 没有以往那么炎热, 而南边沿海地区时常出现高温 预警，一些港岛受到台风影响连续好几天出现黑雨 警报，这些生态变化是这短短几年不常见的，似乎 越来越多无解的现象正逐步瓦解我们最后一点自信 和自我构筑的信念。这种种事件从来都不曾写进当 代年轻人的手机资讯中，相比于这些 “远在天边” 的大火, 隔岸观火成为一种常态, 而毫无血缘关系 的爱豆塌方事件反而被人们乐此不疲的谈及。就在 这不经意间, 气候变暖, 自然灾害频发, 人们在经 历磨难却不自知的状态下被地球无声的下达最后通 牒, 大自然纵使有多么强大的愈合功能都无法承载 人类在长达几个世纪的工业发展中残留的种种。这 一场病毒表演的背后或许只有少数人看到了人类即
将面对的未来。在书《三体》中有一个现实的比喻: “科技革命是人类社会的一种病变, 技术的爆炸性 发展与癌细胞的飞速扩散相当, 最终的结果都是耗 尽有机体的养分, 破坏器官, 导致其寄宿体的死亡”。 这段话虽然看似和艺术没有直接关系，却从中看到 了人类的过分自信和偏见，作为万物的主宰者，生 物链的顶端终将如何与地球和解, 这是我们每个人 应该深思的问题。

人类每经历一次磨难便会产生一次深远的影响, 或许多年后回看疫情, 会发现它已经在不经意间对 社会产生了影响。“当局者迷旁观者清” 这个道理 是真实存在的, 我们今天深陷其中, 但变化却在发 生着, 从艺术产出视角看, 2020 年经过疫情后的艺 术开始回归本体, 艺术家们的创作开始真正重视以 人为本, 对城市更新、公共生存环境和生命存在的 意义的思考比过去更深远与急切。首先, 最切实的 是表达对医护人员的感激之情的公共艺术作品（如 图 1), 由全球公共艺术制作人 Wild in art 创作 的

《Gratitude（感恩）》，这个装置是向疫情期 间不辞辛苦的工作人员表现出的勇于献身的精神和 勇气致敬, 并通过艺术捕捉这一年我们的非凡经历。 在两

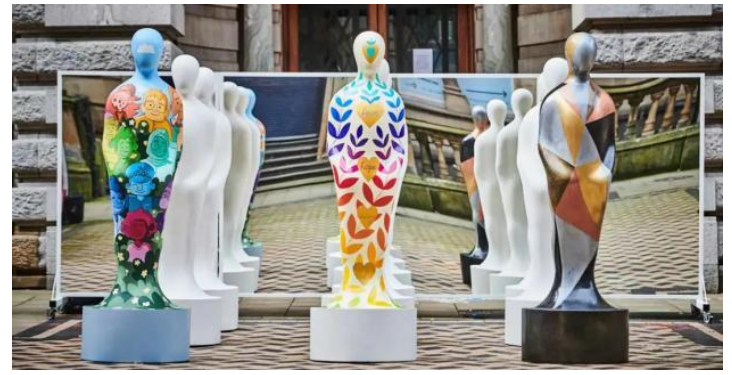

图 1 全球公共艺术制作人 Wild in art 创作的 《Gratitude（感恩）

面镜子的包围下，出现了壮观的倒影，整个公共艺术 装置通过观众沉浸式体验, 感受艺术家的创作用意。

正如古希腊哲学家柏拉图提出的 “我是谁? 我从 哪里来? 我要到哪里去? ” 这一古老的哲学命题, 从 古至今仁人学者都不曾对这个问题停止思考, 作为城 市更新中萌芽的公共艺术也以自己的方式不断追问 并寻找着。进入后疫情时代, 不难发现人们对艺术的 关注点其中之一转变为 “对公共空间新的需求” ，即 把在室内聚集的艺术逐步转向室外空旷之地 (如图 二），如亚特兰大的公共房子装置，旨在为高级博物 


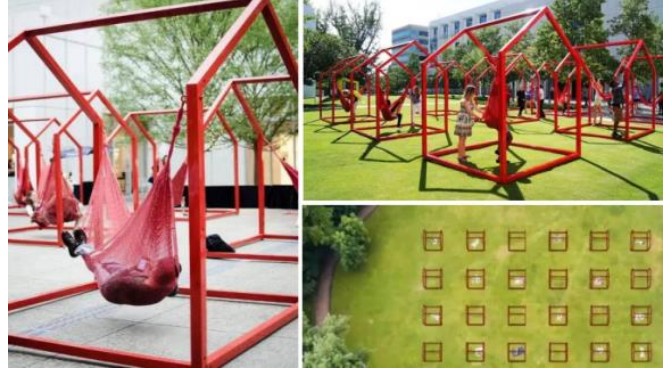

图 2 亚特兰大公共房子装置 -esrawe studio + cadena

馆与周边社区建立联系, 除了可以承载多元文化活 动, 还是个开发社交、展示互动性的良好载体。

疫情对刚刚发展起来的公共艺术影响是直击本 质的, 人们对公共空间的害怕与担心正是我们所思考 的问题, 就是重新构建空间的信任感, 平衡城市中的 个人与城市之间的需求矛盾, 从而唤醒城市活力。温 哥华旅游局在今年三月举办了为期三个月的户外主 题活动《\#LoveVancouver Dance Bubbles》, 活动通 过拉近人与人、人与自然的距离, 为城市重新注入活 力与生机; 同月, 由英国 Paul Cocksedge Studio 创 作而成的《Please Be Seated》来到广州, 别出心裁 地融入新元素, 采用数字投影技术, 创造出一个沉浸 式声光互动空间, 装置满足了距离感的同时希望人们 享受当下, 坐在光陆流离的光影中, 珍惜每一刻快乐 的时光; 今年 5 月, 艺术家 Ekene I jeoma 的互动公 共艺术装置《Breathing Pavilion》在布鲁克林市中 心的阿什兰 300 号广场上展出, 邀请观众随着光线的 变化一起呼吸, 将自己调谐到一个共享的喘息节奏 中, 在经历长期封闭居家的人们对能在自然环境中大 口呼吸都变成了难得。病毒虽然迫使人们改变生活方 式和社交方式, “距离” 这个词将伴随我们不知多久, 因此, 通过设计的多样性改变空间格局, 使拘束变为 自由, 打破隔离带来的单调气氛, 找回城市失落空间 是公共艺术创作在疫情常态化后应该着重注意的。

\section{3. 公共艺术作为愈合灾难中城市的良药价值 意义}

这场疫情虽然掠夺了很多生命, 带来了惨痛的代 价, 但它也从某种意义上将历史格局划分为疫情以前 和疫情之后。疫情以前, 城市空间的发展大多千篇一 律, 地域性不强, 如何将城市文化因子活化, 使功能 性城市向人文城市转变, 是我们在规划城市过程中一 直思考的问题。公共艺术作为城市灵魂的集合体, 不 仅可以激活城市文化, 更能塑造城市精神。可以说公 共艺术的介入是解决城市同质化问题的关键因素之 一。

疫情后, 城市的发展正从停滞状态投入快速复 苏, 审视人与自然的关系, 用公共艺术逐渐安抚城市, 为人们带去慰藉成为新时代人文城市的迫切需求。城 市更新的目的强调在持续不断的更新过程中, 将城市
文化贯彻始终, 推动产品和消费结构升级, 以文化创 意引领历史文脉的传承与延续, 激发城市活力, 再造 城市价值, 同时, 通过相关政策的保驾护航, 最终提 升城市品质，形成可持续发展的人文城市有机更新 ${ }^{[3]}$ 。

公共艺术和其他艺术不同在于更加强调互动性 和大众性, 与城市共生, 相互促进。随着经济的发展, 物质水平到达一定高度, 越来越多对精神的追求充斥 着我们的生活，由此激发了城市规划中对公共空间的 新要求, 这一点, 公共艺术正合理迎合大众需求, 因 此在城市化进程中得到重视。总而言之, 公共艺术性 的创造物和艺术景观作为城市艺术中的重要类型, 主 要承担着改善城市公共环境的职责, 也有着提升区域 艺术格调以及提升区域文化风貌、缓解压力以及创造 巨大经济效益的作用和现实意义 ${ }^{[4]}$ 。

\section{4. 后疫情时代城市更新过程中公共艺术的设 计策略}

国外公共艺术的发展特别是发达国家已趋于饱 和, 而国内公共艺术正随着城市发展进程逐渐盛开, 其进步空间还很大, 多元开放和宽容化的特征足以让 公共艺术立足城市各个角落, 但对于场域特有属性、 共融性及原创性还有待提高。在改进过程中我们应该 多角度多层次出发, 达到平衡。

\section{1 为公共艺术制定文化制度及政策}

社会性是城市公共艺术的重要属性之一, 随着城 市复苏更新, 公共艺术既要加快步伐又应该成熟有序 的发展, 对此, 我们应该向西方学习为公共艺术建立 相关文化制度和政策。因城市公共艺术涉及的领域很 广泛, 需要不同部门共同协作, 以规范化制度来约束 管理, 可以避免公共空间出现杂乱无章及文化场域错 位的问题。除此, 公共艺术还具备公共性原则, 在政 府以规范领导前提下增加公众话语权, 展现艺术的包 容性和参与互动性。

\section{2 多层次多角度发展城市公共艺术}

在人际交往日益淡漠的当代社会, 公共艺术的介 入促进了交流沟通, 增进了共同认知, 对于修复社会 交往的纽带是有益的 ${ }^{[5]}$ 。借助公共艺术表达的思想, 改善城市公共空间景观规划, 潜移默化地融入公众生 活、精神中。

多层次发展公共艺术, 需要将作品与所处地域环 境、当地自然环境, 社会人文气息等有效考量, 细化 可以分为公共艺术作品与周边植物、建筑、设施、道 路, 空间等的调和共生关系。让作品真正发挥城市核 心价值, 在视觉上吸引公众消费, 在内在文化的表达 上与公众产生共鸣, 成为城市情感交流的中心和文化 汇聚的符号象征。多角度发展公共艺术, 需要明确与 公众的合作, 不仅在作品落地后才逐渐与公众相连, 
还需要在设计过程中与公众产生交流, 共同筑造作品 氛围, 真正实现公共艺术与社会的良好沟通与共同实 践。

\section{3 激发设计师的创作潜能}

作为设计师, 除了要保持原创性和独立思考的能 力外, 还应该学会多视角对整个社会不同阶级环境进 行观察, 表达自己内心世界的同时在现社会现状。疫 情常态化后, 人们可以适当走动, 发起一些公共艺术 专题展览是有必要的, 邀请不同设计师共同交流切磋 辩论, 最大限度激发设计师潜能, 创作出更好的作品。 每个设计师在设计过程中与物和空间的对话都是独 一无二的联结, 尊重彼此的同时建立属于自己的设计 理念和风格。保持设计的外在联系和内在独创性。

\section{5. 结语}

好的公共艺术不仅可以解决城市美学问题, 更是 可以重塑城市精神, 强化城市文化特色和性格, 成为 集社会话题、公众参与、提高城市艺术修养, 美学传 播与一体的综合性艺术载体。随着城市复兴、乡村振 兴、文旅融合等各项齐头并进的发展和大众审美标准 的升级, 塑造城市公共艺术成为了一个广泛且重要的
话题。为此我国的艺术设计人员都应该肩负使命, 努 力提升我国城市空间的艺术修复和构建，促进城市发 展水平达到平衡。未来, 随着我国城市的转型, 公共 艺术也将被推向高点, 带动城市走向国际化。

\section{REFERENCES}

[1] Wu Shixin. "Opportunity" for innovation in "crisis" : Development of public art in post-epidemic period [J]. Sculpture, 2020, (5) : 56-59.

[2] Zhang Yujie. Public Art during the Epidemic [J]. Public Art, 2020,(04):74-80.

[3] Qin DI. People-oriented design concept and method in urban organic renewal [J]. Urban development research, 2019,26 (2) : 36-40.]

[4] $\mathrm{Fu}$ Xingye. The Significance of Public Art in contemporary Urban Development [J]. Art Appreciation, 2019,(23):105-106.

[5] Wang Yao. The Intervention of Public Art on Urban Public Space in China [J]. Beauty \& Times (Urban Edition), 2018,(07):65-66. 